\title{
Tumor de Wilms
}

\section{Wilms Tumor}

Pág. 153,162

Recibido: $20-05-2020$

Aceptado: 25-06-2020

Dra. Marcela Kriebel Haehner ${ }^{1}$

Dra. Adriana Zúñiga Villegas ${ }^{2}$

Dr. Daniel Morún Calvo ${ }^{3}$

Dr. Andrey González Chavarría ${ }^{4}$

12,3,4. Médico General, Trabajador Independiente. San José,Costa Rica.

\section{RESUMEN}

El tumor de Willms es el tumor renal más frecuente en la edad pediátrica. Histológicamente se encuentra compuesto por tres tipos de células. Tiene una presentación clínica en la mayoría de los casos esporádica, sin embargo se ha visto asociado a síndromes genéticos. Actualmente tras su diagnóstico y tratamiento, se ha visto que sus principales complicaciones se asocian a los quimioterapeuticos utilizados. En nuestro país se ha evidenciado una tasa de sobrevivencia superior a los países sub desarollados.

\section{PALABRAS CLAVE}

Tumor de Wilms, congenito, riñón, infancia

\section{ABSTRACT}

Willms' tumor is the most frequent renal tumor in pediatric age. Histologically it is made up of three types of cells. It has a clinical presentation in most cases sporadic, however it has been associated with genetic syndromes. Currently after its diagnosis and treatment, it has been seen that its main complications are associated with the chemotherapeutic agents used. In our country, a higher survival rate than in the developed countries has been evidenced.

\section{KEY WORDS}

Wilms Tumor, congenital, kidney, childhood

\section{INTRODUCCIÓN}

El tumor de Wilms representa el $7 \%$ de todos los tumores de la edad pediátrica, siendo el tumor renal más común. En Estados Unidos, se diagnostican alrededor de 500 casos nuevos al año, siendo la incidencia anual aproximadamente de 7 casos por millón de menores de 15 años. Se reporta que dos tercios de los casos se documentan en menores de 5 años y un 95\% antes de los 10 años. Además, la edad promedio 
para el diagnóstico de pacientes con compromiso unilateral es de 43 meses en mujeres y 37 meses en hombres. No obstante, cuando compromete ambos riñones $o$ anormalidades congénitas el diagnóstico se hace a edades más tempranas. El factor de riesgo principal es el grupo etario, siendo el afroamericano con mayor riesgo y el asiático con el menor (1).

El objetivo de esta revisión bibliográfica es una actualización en el tema para un mejor abordaje a esta entidad.

\section{MÉTODOS}

Esta revisión bibliográfica se utilizaron bases de datos como PubMed, Up to Date y Medline cómo buscadores para la epidemiología, fisiopatología, manifestaciones clínicas, diagnóstico y manejo de esta patología. Los criterios de inclusión publicaciones con las palvaras clases "Tumor de Wilms", "congénito","riñón", en el idioma español e ingles. Se revisaron $X$ artículos que contienen puntos esenciales sobre esta entidad en vista del desarrollo del objetivo establecido.

\section{FISIOPATOGENIA}

Seha reportado que el tumor de Wilms es causado por un desarrollo renal anormal, que resulta en proliferación de un blastema metanéfrico sin diferenciación tubular y glomerular normal. Los restos nefrogénicos normalmente ocurren en $1 \%$ y retroceden temprano en la infancia. Estos restos están presentes en el $35 \%$ de riñones con un tumor de Wilms unilateral y en casi el $100 \%$ con afectación bilateral (2).

Estudios han relacionado al tumor de Wilms con una pérdida de la función por mutaciones de varios genes supresores de tumores y genes de transcripción. A pesar de que todavía no se conoce bien la asociación de las mutaciones con la patogénesis del nefroblastoma, se ha vinculado con los cambios en el gen WT1, p53, FWT1, p y el locus 11p15.5 (3).

Adicionalmente, se han estudiado los tumores de Wilms familiares (FWT) que representan de 1 a $2 \%$ de los casos. La herencia parece ser autosómica dominante y no se asocia a mutaciones en el gen WT1 (1)

Aún se desconoce si la presencia de alguno de los genes mencionados afecta la respuesta al tratamiento o si es predictiva del pronóstico.

Es una patología de tumor solitario en la mayoría de sus presentaciones. No obstante, de un 5 a un $7 \%$ se presenta de forma bilateral y alrededor de un $10 \%$ tiene afectación multifocal en un solo riñón. Dentro de su patología, el nefroblastoma incluye quistes, hemorragia y necrosis. Usualmente se encuentra rodeado de una pseudocápsula, lo cual ayuda a diferenciar de otros tumores renales que tienen un borde infiltrativo (1).

Histológicamente, el tumor de Wilms clásico está compuesto por tres tipos de células. Las células del blastema que son células desdiferenciadas encargadas de procesos de proliferación celular y rediferenciación de nuevas estructuras. Las células estromales son células inmaduras que dan origen a el músculo esquelético, cartílago, hueso y grasa. El tercer tipo de células son las epiteliales, las cuales son propiamente de tejido renal que compone los túbulos y glomérulos (4, 5).

A diferencia de las mutaciones genéticas, la histología del nefroblastoma sí está ligado al pronóstico del paciente. La anaplasia, definida cómo la presencia de figuras mitóticas polipoides 
multipolares y un marcado aumento nuclear con hipercromasia, se asocia con un peor pronóstico.

\section{PRESENTACIÓN CLÍNICA}

El tumor de Wilms se presenta solo con una masa abdominal o inflamación en la mayoría de los pacientes (6). Otros síntomas que pueden estar presentes son el dolor abdominal en un 30 a $40 \%$ de los niños, hematuria en un 12 a $25 \%$, fiebre e hipertensión en un $25 \%$ de los casos. Al examen físico, se palpa una masa firme, no distendida y lisa que se encuentra excéntricamente y rara vez cruza la línea media. Un subgrupo de pacientes pueden tener una hemorragia subcapsular, ellos podrían presentar un rápido crecimiento abdominal, anemia, hipertensión y en algunas circunstancias fiebre. A pesar de que los pulmones son el primer sitio de metástasis, muy raramente los pacientes van a aquejar síntomas respiratorios (1).

Una vez que se sospeche el tumor de Wilms, la palpación abdominal debe ser cuidadosa debido a que puede causar una ruptura de la cápsula renal. Esto puede resultar en un derrame tumoral que aumenta el estadio del tumor y la necesidad de una terapia más intensiva (1).

El tumor de Wilms se asocia a síndromes y anomalías congénitas previamente mencionadas, por lo que al examinar al paciente se debe incluir una evaluación para asociar o descartar que esto suceda. Durante el examen físico hay que buscar anomalías como lo es la aniridia, hemihipertrofia o alteraciones genitourinarias (1).

\section{Evaluación Diagnóstica}

El paciente que llega a la consulta se debe de abordar con buena historia clínica incluyendo antecedentes heredofamilares de neoplasia y un examen físico completo. En el caso tumor de Wilms, el diagnóstico definitivo se realiza mediante biopsia o una excisión quirúrgica debido a que se requiere la confirmación histológica. Cualquier niño al que se le sospeche un nefroblastoma, debe ser referido a un hospital pediátrico para la evaluación, diagnóstico y tratamiento del mismo $(4,7)$.

Los exámenes de laboratorio se realizan de rutina para descartar otras patologías (4) Por ejemplo, examen general de orina se colecta para ver si el paciente tiene proteinuria, debido a que esto puede pasar en el Síndrome de DenysDrash y en esclerosis mesangial. Se toman pruebas de función hepática para descartar metastasis al hígado. El calcio sérico se puede elevar en un tumor rabdoide del riñón o un nefroma mesoblastico congénito. Por último, las pruebas de coagulación son importantes para estudiar si el paciente tiene un trastorno de la coagulación que afecte a la hora de la cirugía y poder corregirlo en caso necesario. Dentro de dichas pruebas, de debe incluir el factor de von Willebrand, en especial si el paciente tiene historia de sangrado 0 tumores hipervascularizados, porque en 4 a $8 \%$ de los pacientes con un trastorno de este factor presentan un tumor de Wilms al diagnóstico (1).

Uno de los pilares en el diagnóstico son los estudios de imagen abdominales, ya que con ellos de puede diferenciar un tumor de Wilms de otras masas abdominales. Otra información importante es confirmar la presencia y función del riñón colateral, sí hay un tumor bilateral, el tamaño del tumor y la presencia de metástasis (3).

El estudio inicial que se realiza es un ultrasonido

\section{(ㄷ) $(1) \Theta \theta$}


abdominal en el cual se puede detectar hidronefrosis y enfermedad renal multiquística. En caso de un tumor renal permite realizar un ultrasonido Doppler para observar si hay infiltración tumoral de la vena renal y la vena cava inferior y así evaluar la permeabilidad del flujo sanguíneo (1).

La tomograífa axial computarizada (TAC) con medio de contraste o una resonancia magnética (RM) se recomienda para evaluar posteriormente la naturaleza y extensión de la masa abdominal (1).

Estudio se imagen de tórax se realiza para determinar si existe metástasis a pulmones. En caso de que salgan lesiones sospechosas, se debe proceder a un TAC de tórax. Si se revela un nódulo sospechoso se le tiene que realizar una biopsia (1).

\section{Diagnósticos Diferenciales}

Los diagnósticos diferenciales del tumor de Wilms incluye otros tumores renales como el neuroblastoma. El más fácil de diferenciar es el neuroblastoma, debido a que en un ultrasonido, TAC o RM se distingue si es tejido renal o no, sin embargo siempre se requiere histología. El sarcoma de células claras del riñón es el segundo más común en la edad pediátrica. Este sarcoma tiene un peor pronóstico que el tumor de Wilms con aumento en la recaída y mortalidad $(1,8)$.

\section{Estadiaje}

El estadiaje del tumor de Wilms se basa en la extensión anatómica del tumor. Esta no toma en cuenta factores externos como genética ni histología. Entre las etapas más altas, peor el pronóstico por lo que el abordaje debe ser más agresivo. Actualmente existen dos sistemas que se utilizan $(1,8)$.

EI National Wilms Tumor Study (Tabla 1) es el que se usa en Estados Unidos y Canadá y se basa en la evaluación quirúrgica previo a administrar otros tratamientos como la quimioterapia. En cambio, el de la Sociedad Internacional de Oncología Pediátrica (Tabla 2), utilizado en Europa, se basa en una evaluación quirúrgica posterior a la administración de quimioterapia $(1,8)$.

Tabla 1. Sistema NWTS (National Wilms Tumor Studies).

\begin{tabular}{|c|c|}
\hline Etapas & Características \\
\hline Etapa I & $\begin{array}{l}\text { - Tumor limitado al riñón. } \\
\text { - Se reseca completamente con una cáp- } \\
\text { sula intacta sin evidencia de afectación } \\
\text { de los vasos del seno renal o tumor en o } \\
\text { más allá de los márgenes de la resec- } \\
\text { ción. } \\
\text { - No hay ruptura o biopsia previa. }\end{array}$ \\
\hline Etapa II & $\begin{array}{l}\text { - El tumor se extiende más allá del riñón, } \\
\text { pero se reseca en su totalidad. } \\
\text { - Su extensión incluye invasión del tejido } \\
\text { blando del seno renal, o compromiso de } \\
\text { los vasos sanguíneos más allá del pa- } \\
\text { rénquima renal pero dentro de la mues- } \\
\text { tra resecada sin ruptura. }\end{array}$ \\
\hline Etapa III & $\begin{array}{l}\text { - Posterior a la cirugía, hay remanente } \\
\text { tumoral, pero está limitado al abdomen. } \\
\text { - Ruptura o biopsia del tumor. }\end{array}$ \\
\hline Etapa IV & $\begin{array}{l}\text { Existe metástasis hematógena o en gan- } \\
\text { glios linfáticos más allá de la región ab- } \\
\text { dominlpelvica. }\end{array}$ \\
\hline Etapa V & $\begin{array}{l}\text { - Tumor bilateral a la hora del diagnóstico. } \\
\text { - Cada uno se clasifica por separado. }\end{array}$ \\
\hline \multicolumn{2}{|c|}{$\begin{array}{l}\text { FUENTE: Adaptado de la sección "Staging" del artículo } \\
\text { "Presentation, diagnosis, and staging of Wilms tumor", Up } \\
\text { to date. }\end{array}$} \\
\hline
\end{tabular}


Tabla 2. Estadiaje de la Sociedad Internacional de Oncología Pediátrica (SIOP).

\begin{tabular}{|c|c|}
\hline Etapas & Características \\
\hline Etapa 1 & $\begin{array}{l}\text { - Tumor es resecado en su totalidad y limi- } \\
\text { tado al rinón o con una pseudocápsula fi- } \\
\text { brosa. } \\
\text { - Se puede encontrar en la cápsula renal, } \\
\text { pélvis sin involucrar útero y vasos intrare- } \\
\text { nales. } \\
\text { - No hay evidencia de afectación de los va- } \\
\text { sos del seno renal o tumor en o más allá } \\
\text { de los márgenes de resección. }\end{array}$ \\
\hline Etapa & $\begin{array}{l}\text { - El tumor se extiende más allá del riñón o la } \\
\text { pseudocápsula fibrosa, pero se reseca por } \\
\text { completo sin evidencia de restos en los } \\
\text { márgenes de resección o más allá de } \\
\text { ellos. } \\
\text { - La infiltración tumoral que se reseca com- } \\
\text { pletamente puede involucrar el seno renal, } \\
\text { vasos sanguíneos o ganglios linfáticos. }\end{array}$ \\
\hline
\end{tabular}

\begin{tabular}{|l|c|}
\hline Etapas & Características \\
\hline Etapa 3 & $\begin{array}{l}\text { - Posterior a la cirugia, quedan residuos de } \\
\text { tumor, pero limitados al abdomen. }\end{array}$ \\
\hline Etapa 4 & $\begin{array}{l}\text { - Metástasis hematológica y en ganglios } \\
\text { linfáticos más allá de la región abdo- } \\
\text { minopelvica. }\end{array}$ \\
\hline Etapa 5 5 & - Tumor bilateral a la hora del diagnóstico. \\
\hline $\begin{array}{l}\text { FUENTE: Adaptado de la sección "Staging" del artículo } \\
\text { "Presentation, diagnosis, and staging of Wilms tumor", Up } \\
\text { to date. }\end{array}$ \\
\hline
\end{tabular}

\section{PRONÓSTICO}

Se conoce que existen factores pronósticos que se asocian a un alto riesgo de recurrencia o muerte a la hora del diagnóstico (4). Estos son la histología del tumor, el estado del tumor, los marcadores moleculares y genéticos, y edad del paciente (mayor de 2 años). Con respeto a la histología del tumor, la presencia de anaplasia se considera el factosrpredictor más importante de resultados adversos $(5,9)$. La histología tipo blastemal también se asocia a un desenlace desfavorable; sin embargo, no tiene diferencia significativa en cuanto al pronóstico como tal.

El pronóstico basado en los dos diferentes estadiajes del tumor, tanto del NWTS y SIOP, entre mayor etapa, peor es el pronóstico del paciente. Se ha reportado que varios marcadores moleculares y genéticos parecen ser predictivos del resultado como lo son : la pérdida de heterocigocidad y ganancia de $1 \mathrm{q}$ y perfiles de expresión genética que igualmente tienen un factor negativo en los pacientes. Los pacientes menores de 24 meses generalmente tienen menos riesgo de recaer en la enfermedad; asimismo, este factor predictor positivo se ha reducido a medida que se mejora el tratamiento y manejo (9).

\section{TRATAMIENTO}

Los pacientes diagnosticados con tumor de Wilms son tratados en base a protocolos, terapia basada en el riesgo de cada uno, la etapa inicial y la clasificación histológica. No obstante, los regímenes que se basan en cirugía, quimioterapia y radioterapia son los que han resultado con mejoras significativas en la tasas de supervivencia general a cinco años, siendo cercanas al $90 \%(9,10)$.

EI NWTS tiene dos ventajas, primero permite una evaluación temprana y precisa sobre el tipo histológico y extensión del tumor de Wilms. En segundo lugar, previene a pacientes con tumores benignos recibir quimioterapia innecesaria. En comparación, con el SIOP, el volumen de tumor se reduce con la quimioterapia prequirúrgica

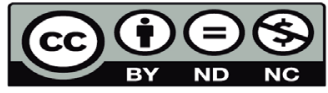


haciendo que la excisión del tumor sea más sencilla. Esto parece que reduce la necesidad de la radioterapia local (9).

Los protocolos del NWTS se basan en la resección quirúrgica como tratamiento inicial para el tumor de Wilms. En etapas I y II se realiza el procedimiento quirúrgico y posteriormente se le dan 19 semanas de vincristina y actinomicina. Los pacientes pueden ser manejados solo con la nefrectomía parcial si cumplen los criterios de tumores de muy bajo riesgo, los cuales son tener menos de 2 años, etapa I con histología favorable y que el tumor pese menos de $550 \mathrm{~g}$. $\mathrm{Si}$ el paciente tiene marcadores moleculares de alto riesgo, se le adiciona doxorrubicina como quimioterapéutico.

En la etapa III, se les dan 25 semanas de quimioterapia con vincristina, actinomicina y doxirubinia posterior a la cirugía. En caso de que se haya involucrado los nódulos linfáticos o el peritoneo se les da terapia con radiación. En la etapa IV, se trata igual que la III, pero en caso de que haya metástasis a pulmón se le administra radioterapia generalizada al lado afectado si no resuelve con el tratamiento inicial. Si otras metástasis se administra radioterapia a la regiones locales $(9,10)$.

El abordaje según los protocoles del SIOP se basan en 4 semanas de quimioterapia prequirúrgica seguido de la resección quirúrgica, $y$ en caso de ser necesario quimioterapia posterior al procedimiento. En pacientes que no tengan evidencia de metástasis, se les administra vincristina y actinomicina. En caso de que sí presenten la enfermedad diseminada más allá de la región abdominopélvica se les da una terapia triple, con las dos quimioterapias antes mencionadas más doxorrubicina por 6 semanas.
En este sistema existen subclasificaciones de las etapas. En la etapa I, si es de bajo riesgo no requiere quimioterapia 0 radioterapia posterior a cirugía. En riesgo intermedio se les brinda quimioterapia posterior a la cirugía por 4 semanas más; y en el riesgo alto esta se les da por 27 semanas adicionales sin radioterapia. En la etapa II, la terapia en el riesgo bajo e intermedio consiste de quimioterapia postoperatoria con vincristina y actinomicina por 27 semanas sin radiación. Si es de alto riesgo, se les brinda tratamiento con quimioterapia por 34 semanas posterior a la cirugía con doxorrubicina, ciclofossamida, carboplatino y etoposido, con radioterapia adicional.

La etapa III, se aborda igual que en el bajo y mediano riesgo de la etapa II. En la etapa III, a el riesgo intermedio se dan 27 semanas de quimioterapia (vincristina y actinomicina) y radiación. Al alto riesgo se trata al igual que el alto riesgo de la etapa II. La radiación del flanco se utiliza para pacientes con enfermedad local en etapa III. En la etapa IV el esquema cambia un poco. Si es riesgo bajo o intermedio con histología que responde completamente se trata con 27 semanas de quimioterapia sin radiación a pulmón. En el riesgo bajo o intermedio con histología que no responde completamente o alto riesgo, se da quimioterapia (doxorrubicina, ciclofossamida, carboplatino y etoposido) por 34 semanas asociada a radiación $(9,10)$.

En caso de niños con tumores bilaterales, lo que equivale a la etapa $V$ en cualquiera de los protocolos, el manejo es complicado. El objetivo es tratar ambos tumores adecuadamente conservado la función renal que se pueda. Se les da una terapia intensiva al diagnóstico para intentar reducir el tamaño de los tumores y así facilitar la cirugía (9). 


\section{COMPLICACIONES}

Las complicaciones tempranas del tumor de Wilms se relacionan a al tratamiento e incluye los efectos adversos de la quimioterapia y la complicaciones quirúrgicas como la obstrucción intestinal, hemorragia e infección de la herida (9).

A largo plazo, las complicaciones dependen de la intensidad y la cantidad de terapia intensiva que hayan recibido los pacientes. La afectación renal, la cardiotoxicidad dada por el uso de doxorrubicina, la cual puede causar una insuficiencia cardíaca y aumento de la postcarga del ventrículo izquierdo. La vincristina y actinomicina se relacionan a la hepatotoxicidad (9).

\section{TUMOR DE WILMS EN COSTA RICA}

En el 2018 se realizó en Costa Rica un estudio con el objetivo de describir las características de los niños diagnosticados con el tumor de Wilms entre 1991 y 2011 y los diversos tratamientos que recibieron en el Servicio de Oncohematología del Hospital de Niños. En este análisis, siendo el más reciente en el país, se revisaron 69 expedientes de los cuales el $62 \%$ eran mujeres y un $38 \%$ hombres. Parte del propósito de este estudio descriptivo, retrospectivo, fue que en Costa Rica, se cuenta con una experiencia de más de 20 años en el manejo de este tumor, sin embargo, hasta la fecha no existían otras investigaciones que analizaran los datos clínicos de los pacientes, su evolución o la sobrevida de estos al tratamiento empleado (11).

El diagnóstico se dio en $30 \%$ en una edad de 0 a 24 meses e igualmente de 25 a 48 meses. La manifestación clínica más prevalente fue una masa abdominal (55\%), seguido de fiebre (12\%) y hematuria $(12 \%)$. Solo uno de los pacientes presentó hipertensión. Así mismo, dos de los niños consultaron por dolor abdominal después de un trauma y en otros dos se sospechó una apendicitis aguda al inicio. Por otro lado, en 3 casos el tumor fue hallado incidentalmente en estudios por apéndices preauriculares y pólipos rectales (11).

Elestadiolll se presentó de manera más frecuente con un $32 \%$. Dentro de las comorbilidades asociadas, 2 pacientes tenían síndrome de DenysDrash, uno con hemihipertrofia y uno con el síndrome que incluye tumor de Wilms, aniridia, malformaciones genitourinarias y retraso mental (11).

Respecto al tratamiento, un $69,6 \%$ tuvo respuesta completa a la quimioterapia y $8,7 \%$ una respuesta parcial. Un $20 \%$ cursó con fallo al tratamiento. Además, 18 niños presentaron metástasis; $26 \%$ a pulmón y un $4.3 \%$ a hígado. La sobrevida global fue de $73,3 \%$, con un promedio de seguimiento de 7,4 años desde el diagnóstico, y la sobrevida libre de enfermedad fue del $69 \%$ (11).

Un hallazgo interesante fue que en nuestro país se presentó el diagnóstico en hombres más tardíamente que en mujeres; no obstante, se ha descrito que es de manera inversa. Al realizar la comparación de la sobrevida de estos pacientes costarricenses con los de países más desarrollado como Estados Unidos y Europa, es menor en Costa Rica pero es superior a la descrita en la literatura en países subdesarrollados como el nuestro (11).

La evidencia científica presentada permitió conocer de maneja objetiva la respuesta al tratamiento aplicado en el Hospital de Niños para el Tumor de Wilms, con resultados iniciales 
favorables. Al mismo tiempo, da a relucir las características de los niños costarricenses y su similitud en comparación con reportes internacionales (11).

\section{CONCLUSIÓN}

En conclusión, el tumor de Wilms es el tumor renal más frecuente en la infancia, especialmente en menores de 15 años. Usualmente es de origen esporádico pero también se puede presentar por predisposición genética o mutaciones en los genes de los pacientes. A pesar de llevar un largo tratamiento, por lo general el pronóstico es muy favorable en aquellos pacientes que se detectan a tiempo o los que se les da un correcto seguimiento. Costa Rica no es un país con baja prevalencia de la enfermedad; sin embargo, los protocolos empleados en el Hospital Nacional de Niños han causado un impacto positivo en el manejo y sobrevida de los pacientes.

\section{BIBLIOGRAFÍA}

1. Chintagumpala, M., \& Muscal, J. A. (2019, June). Presentation, diagnosis, and staging of Wilms tumor. Retrieved November 30, 2019, from Up to Date, https://ezproxy.ucimed.com:2055/ contents/presentation-diagnosis-and-staging-of-wilms-tumor?search=wilms tu-mot\&source=search_result\&selectedTitle=1 95\&usage_type=default\&display_rank=1.

2. Vasallo, P. M., Pérez, J. J. G., Gallego, E., Molina, J. C. F., \& Lecuona, E. (1996, February 1). Desarrollo renal: Factores de crecimiento, celularidad y transporte de sodio y potasio. Retrieved December 5, 2019, from https://www.revistanefrologia.com/es-desarrollo-renal-factores-crecimiento-celularidad-transporte-sodio-potasio-articulo-X021169959600823X.

3. Davidoff, A. M. (2009, June). Wilms Tumor. National Institute of Health. PDF. Tennessee, USA.

Tratamiento del tumor de Wilms y otros tumores renales infantiles (PDQ®)-Versión para pa-

4. cientes. (n.d.). Retrieved from https://www.cancer.gov/espanol/tipos/rinon/paciente/tratamiento-wilms-pdq.

Cajaiba , M. M., \& Reyes-Múgica, M. M. (2007, October). Tumores renales de la infancia y ado-

5. lescencia asociados a anomalías cromosómicas. PDF.

Fariñas, M., Amparo, Hamadou, Blanco, P., Manuel, Landeau, C., ... Ibrahim, L. (n.d.). Caracterís-

6. ticas clinicoterapéuticas de niños y adolescentes con neoplasias renales. Re-trieved December 4, 2019, from http://scielo.sld.cu/scielo.php?script=sci_arttext\&pid=S1029-30192015000700001.

Tumor de Wilms. (2018, March 6). Retrieved from https://www.mayoclinic.org/es-es/diseases-conditions/wilms-tumor/symptoms-causes/syc-20352655. 
7. Illadea, L., Hernandez-Marques, C., Cormenzanaa, M., Lassalettaa, Á., Andión Catalán, M., Ruanoa, D., ... Madero López, L. (n.d.). Tumor de Wilms: revisión de nuestra experiencia en los últimos 15 años. PDF. España.

8. Chintagumpala, M. A., \& Muscal, J. A. (2019, June). Treatment and prognosis of Wilms tu-mor. Retrieved December 6, 2019, from Up to Date, https://ezproxy.ucimed.com:2055/contents/presentation-diagnosis-and-staging-of-wilms-tumor?search=wilms tu-mot\&source=search_result\&selectedTitle=1 95\&usage_type=default\&display_rank=1.

9. Metzger, M. L., \& Dome, J. S. (n.d.). Current Therapy for Wilms' Tumor. PDF. Tennessee, USA.

10. Quirós Mata, M., \& Gamboa-Chavez, A. Y. (2018). Tumor de Wilms en niños de Costa Rica. PDF. San José, Costa Rica. 